\title{
Non-thrombotic ischemic cerebrovascular events related to the use of tyrosine kinase inhibitors in patients with cancer: high-resolution magnetic resonance imaging findings
}

\author{
Jae Eun Sim, Jeongho Park, Oh Young Bang \\ Department of Neurology, Samsung Medical Center, Sungkyunkwan University School of Medicine, Seoul, Korea
}

Received: July 2, 2021

Revised: July 20, 2021

Accepted: July 23, 2021

Corresponding author:

Oh Young Bang

Department of Neurology,

Samsung Medical Center,

Sungkyunkwan University

School of Medicine, 81 Irwon-ro,

Gangnam-gu, Seoul 06351, Korea

Tel: +82-2-3410-3599

E-mail: neuroboy50@naver.com

\begin{abstract}
The targeted cancer therapies that have been introduced in recent years are directed against cancer-specific molecules and signaling pathways, and thus, they have limited nonspecific toxicities. However, major vascular events, including stroke, are common in patients receiving tyrosine kinase inhibitors (TKIs). Inhibition of vascular endothelial growth factor receptor by some TKIs probably explains such events. Moreover, venous and arterial thromboses, atherosclerosis, and bleeding have been reported. Ischemic lesions can also occur due to impaired angiogenesis or endothelial dysfunction. However, the exact mechanisms of arterial stroke in patients with cancer receiving TKIs are unknown. Here, we have report two cases of non-thrombotic ischemic cerebrovascular events related to TKIs and described the high-resolution magnetic resonance imaging findings.
\end{abstract}

Keywords: Angiogenesis; Atherosclerosis; Neoplasms; Stroke; Tyrosine kinase inhibitors
This is an Open Access article distributed under the terms of the Creative Commons Attribution Non-Commercial License (https:// creativecommons.org/licenses/ by-nc/4.0/).

\section{INTRODUCTION}

Cerebrovascular disease commonly occurs in patients with cancer. Active cancer and chemotherapy promote hypercoagulability, thus leading to stroke [1]. Although the control of active cancer can reduce the risk of cancer-related stroke, cancer chemotherapy may be associated with an increased risk of arterial events [2]. Chemotherapeutic agents such as cisplastin, methotrexate, and L-asparaginase, may enhance thrombin generation and increase the risk of stroke $[2,3]$. However, mechanisms of stroke related to chemotherapy may differ depending on the types of therapy used [3]. With recent introduction of targeted therapy, such as tyrosine kinase inhibitor (TKI) and angiogenesis inhibitor therapy, the subtypes of stroke related to cancer therapy should also be changed accordingly.

The recently introduced targeted therapies for cancer are directed against cancer-specific molecules and signaling pathways, and thus, they have fewer nonspecific toxicities. Tyrosine 
kinases are especially important targets because they play vital roles in the modulation of growth factor signaling. Multitargeted TKIs against vascular endothelial growth factor receptor (VEGFR) 1, 2, and 3; fibroblast growth factor receptor (FGFR) 1-4; platelet-derived growth factor receptor (PDGFR) $\alpha$; RET; and KIT have therapeutic efficacy in some tumors.

TKIs are associated with stroke and transient ischemic attack (TIA) [4]. A meta-analysis of major adverse events in patients treated with TKIs found that arterial events are common [5]. In a randomized trial involving patients with progressive thyroid cancer, arterial, or venous thromboembolic events occurred more frequently in the multi-targeted TKI group than in the placebo group [6]. However, the underlying mechanisms of arterial stroke in patients with cancer receiving TKIs are unknown. Here, we have reported two cases of non-thrombotic stroke related to TKIs and presented the associated high-resolution magnetic resonance imaging (MRI) findings. All patients were provided written informed consent.

\section{CASE REPORTS}

\section{Case 1}

A 51-year-old man visited our hospital with two episodes of dysarthria, each lasting $<1$ minute. He had a history of hy- perglycemia, for which he was not receiving medications, but no other vascular risk factors. He had been receiving a TKI (nilotinib) for chronic myeloid leukemia for 44 months. MRI of the brain showed no demonstrable acute infarcts, and time-of-flight magnetic resonance angiography revealed stenosis of the left middle cerebral artery (MCA). Work-up for potential cardiac embolic events, including 24-hour telemonitoring and echocardiography, revealed negative findings. The D-dimer level was $0.27 \mu \mathrm{g} / \mathrm{mL}$.

Brain MRI showed a focal enhancing plaque in the left MCA (Fig. 1). Aspirin and high-intensity statin (atorvastatin $80 \mathrm{mg}$ ) were initiated, and nilotinib was switched to dasatinib. The patient's condition was stable, and no recurrence was noted during the 1-year follow-up.

\section{Case 2}

A 64-year-old man with hypertension and hepatocellular carcinoma ( $\mathrm{HCC}$ ) with peritoneal seeding presented with recurrent cerebral infarcts four times over a period of 3 months (Fig. 2A). He had been receiving a multi-targeted TKI (lenvatinib) for HCC for 7 months. At presentation he experienced left-sided hypoesthesia and weakness, and MRI of the brain showed bilateral thalamic infarctions. The patient was treated with aspirin. One month later, he visited the emergency

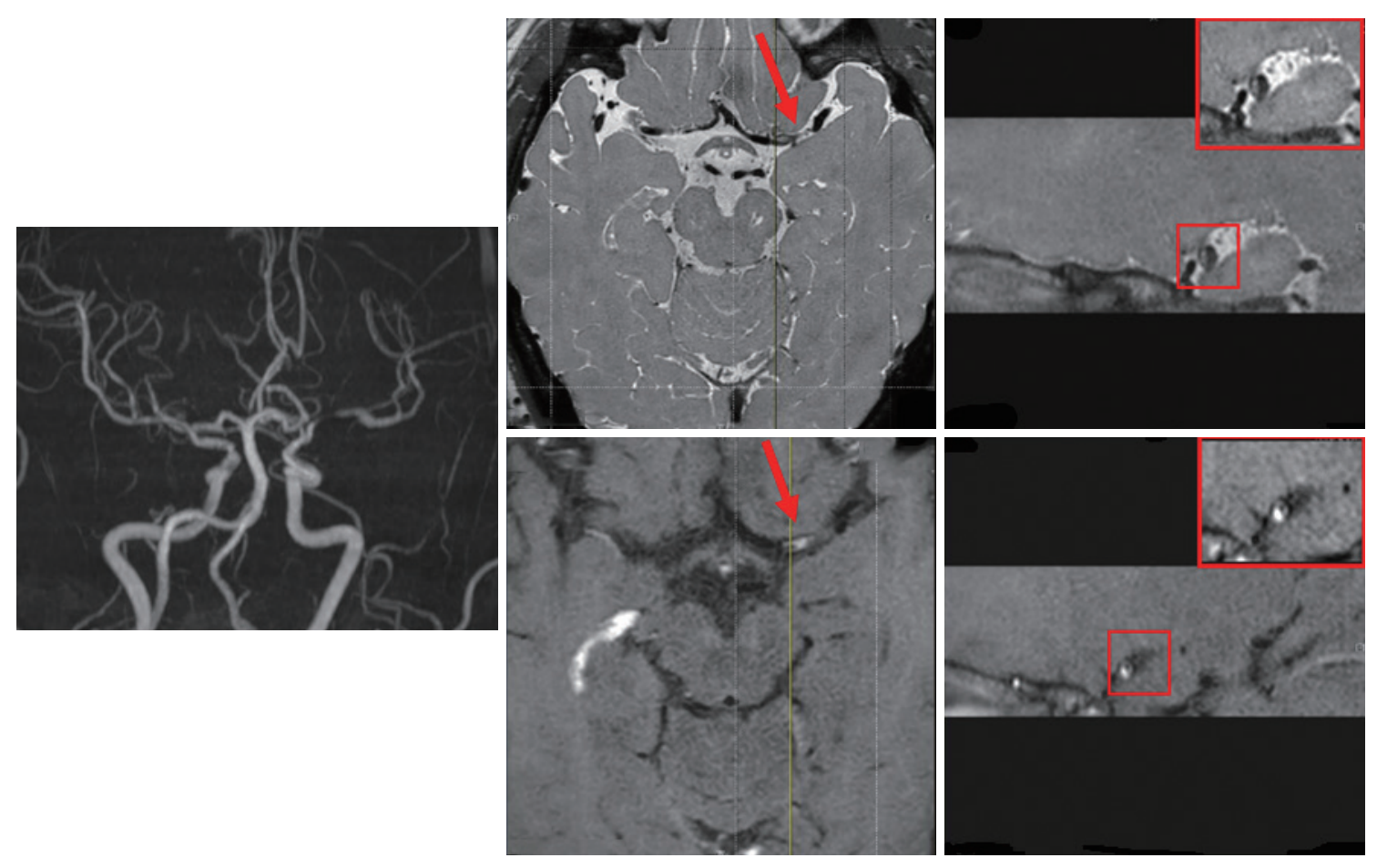

Fig. 1. Magnetic resonance angiography revealing left middle cerebral artery stenosis. T1 weighted high-resolution brain magnetic resonance image showing focal enhanced plaque (red arrows) in the left middle cerebral artery. 
room for abdominal pain and was diagnosed with pancreatitis. He also complained of worsening left-sided weakness. MRI of the brain showed infarcts in the right posterior limb of the internal capsule and basal ganglia. After 3 weeks, he experienced worsening of left-side hypoesthesia and dysarthria, and a new diffusion-restricted lesion was observed in the right centrum ovale. After 7 weeks, he complained of new right-hand weakness despite treatment with dual antiplatelet agents (aspirin and clopidogrel). MRI of the brain showed a new lesion in the left centrum ovale. Work-up for potential cardiac embolic events, including 24-hour telemonitoring and echocardiography, revealed negative findings. Cerebrospinal fluid examination did not show signs of leptomeningeal seeding and vasculitis. The D-dimer level was $0.39 \mu \mathrm{g} / \mathrm{mL}$. High-resolution MRI revealed a focal enhancing plaque in the right proximal MCA, suggesting unstable atherosclerotic plaque (Fig. 2B).

\section{DISCUSSION}

To the best of our knowledge, this is the first report of highresolution MRI-proven atherosclerotic stroke or TIA related to the use of TKIs. TKI-related adverse events usually devel- op after $>1$ to 2 years of treatment $[4,6]$. However, a shorter period of exposure to a multi-targeted TKI could be associated with cerebral infarcts, as seen in case 2. Moreover, very frequent strokes shortly after initiation of TKIs suggest a causal relationship between TKI use and cerebrovascular events.

The pathophysiology of TKI-related arterial events is unclear, but it is thought to be multifactorial. TKI induce changes in vessel wall homeostasis and impair proliferation of endothelial cells. Lenvatinib is an oral inhibitor of VEGFR 1, 2, and 3; FGFR 1-4; PDGFR $\alpha$; RET; and KIT. It primarily inhibits vascular endothelial growth factor (VEGF)-driven angiogenesis (Fig. 3) [3]. Similarly, nilotinib is associated with accelerated atherosclerosis [7]. Nilotinib inhibits several additional kinase targets, including VEGFR 2, in tumor cells (Fig. 3). Nilotinib exerts direct pro-atherogenic and antiangiogenic effects on vascular endothelial cells, including suppression of endothelial cell proliferation, migration, and tube formation. The reason we chose dasatinib over nilotininb in case 1 was because nilotinib, a second-generation BCR/ABL1 TKI, has a higher rate of arterial vascular adverse events than dasatinib [4].

Similarly, angiogenesis inhibitors (such as bevacizumab, a VEGF-A inhibitor), which interfere with these normal process-
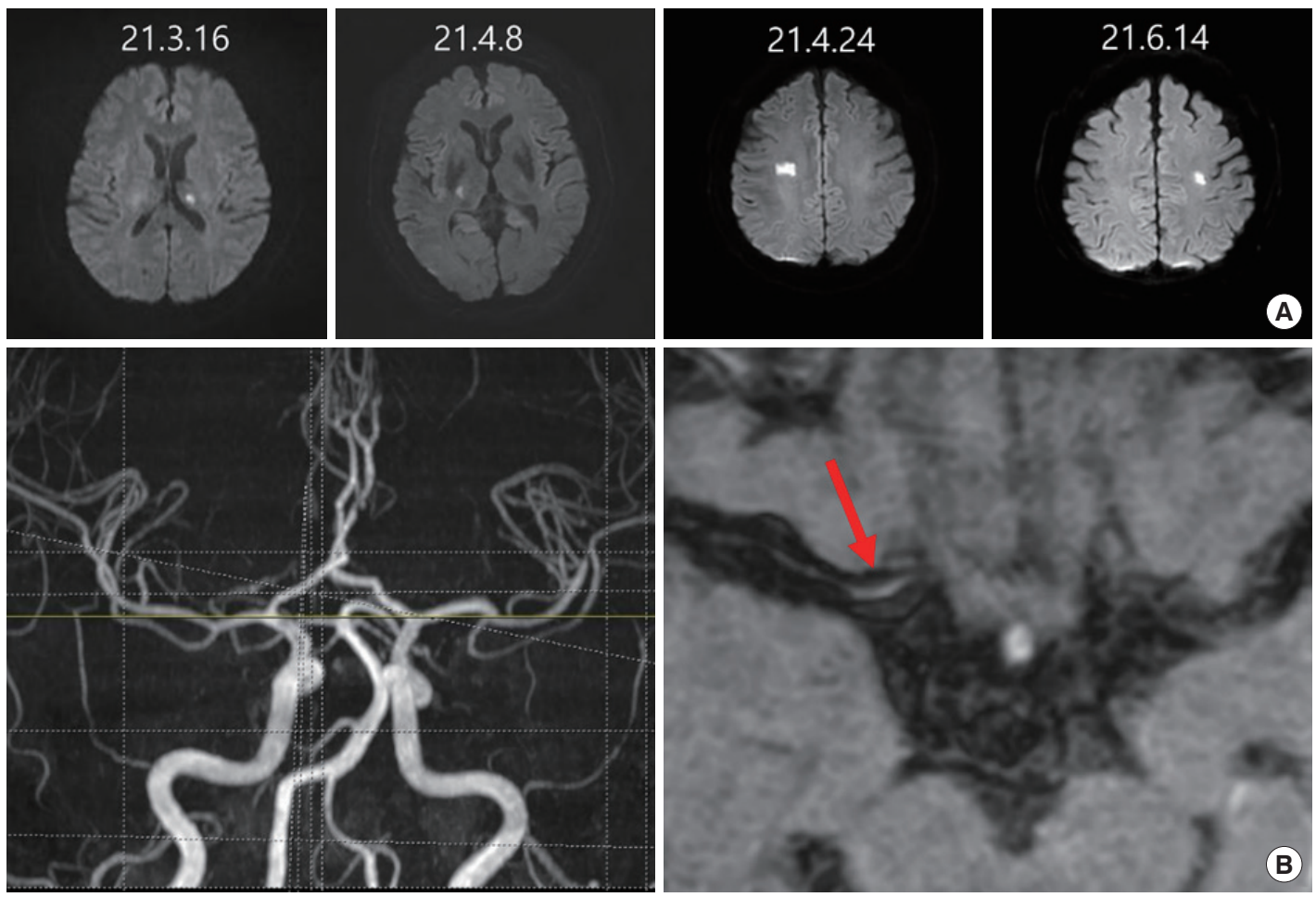

Fig. 2. (A) Diffusion-weighted magnetic resonance axial images of the brain showing four consecutive cerebral infarcts over a period of 3 months (year-month-day). (B) T1 weighted high-resolution brain magnetic resonance image showing focal enhanced plaque (red arrow) in the proximal right middle cerebral artery. 
PRECISION AND FUTURE MIEDICINE

Non-thrombotic ischemic cerebral events related to TKI

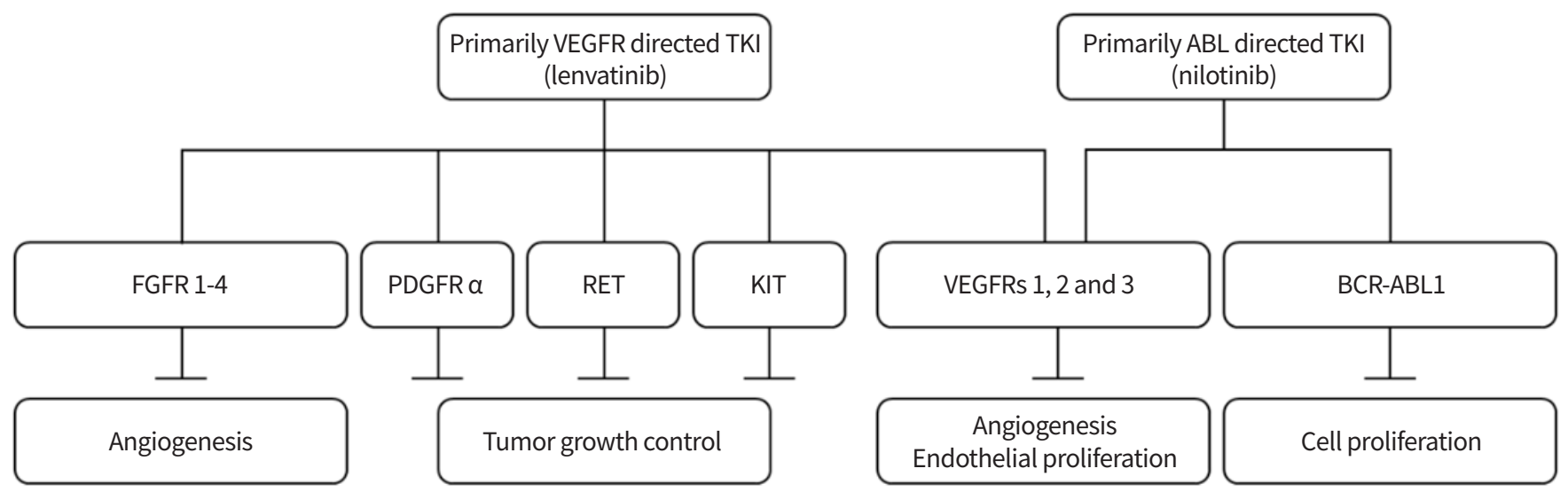

Fig. 3. Mechanism of action of lenvatinib and nilotinib. Lenvatinib is a tyrosine kinase inhibitor (TKI) primarily directed against vascular endothelial growth factor receptor (VEGFR). It inhibits VEGFR 1, 2, and 3; fibroblast growth factor receptor (FGFR) 1-4, platelet-derived growth factor receptor (PDGFR) $\alpha$; RET; and KIT. Nilotinib is a TKI primarily directed against BCR-ABL1. It inhibits BCR-ABL1 and VEGFR2.

es, may predispose to conditions such as stroke, coronary artery disease, and peripheral arterial disease [8]. In contrast, epidermal growth factor receptor (EGFR) inhibitors attenuate atherosclerosis via decreasing inflammation and oxidative stress [9]. Therefore, EGFR inhibitors could be a safer and effective treatment option in patients with lung cancer and cerebral infarction [10].

In conclusion, non-thrombotic stroke due to accelerated intracranial atherosclerosis may be an adverse effect of TKI therapy, especially VEGFR inhibitor therapy, in patients with cancer, probably due to their effects on angiogenesis. Our cases provide supporting evidence of accelerated atherosclerosis related to angiogenesis that inhibited chemotherapy. Because cancer and stroke share risk factors (such as smoking and obesity), patients with cancer could be prone to atherosclerosis. Therefore, newer targeted therapies should be used with caution, especially in patients with multiple vascular risk factors or established atherosclerosis. Therapeutic strategies for atherosclerosis, such as intensive statin therapy, can be useful in these patients, but their efficacy in clinical setting requires further research.

\section{CONFLICTS OF INTEREST}

Oh Young Band has been editorial board of P\&FM since January 2017. He was not involved in the review process of this case report.

\section{ACKNOWLEDGMENTS}

This study was supported by the National Research Founda- tion of Korea grant (No. 2018R1A2B2003489).

\section{ORCID}

Jae Eun Sim https://orcid.org/0000-0002-4583-4805

Jeongho Park https://orcid.org/0000-0003-3115-462X

Oh Young Bang https://orcid.org/0000-0002-7962-8751

\section{AUTHOR CONTRIBUTIONS}

Conception or design: JES, OYB.

Acquisition, analysis, or interpretation of data: JES, JP.

Drafting the work or revision: JES, OYB.

Final approval of the manuscript: OYB.

\section{REFERENCES}

1. Bang OY, Chung JW, Lee MJ, Seo WK, Kim GM, Ahn MJ, et al. Cancer-related stroke: an emerging subtype of ischemic stroke with unique pathomechanisms. J Stroke 2020;22: 1-10.

2. Li SH, Chen WH, Tang Y, Rau KM, Chen YY, Huang TL, et al. Incidence of ischemic stroke post-chemotherapy: a retrospective review of 10,963 patients. Clin Neurol Neurosurg 2006;108:150-6.

3. Campia U, Moslehi JJ, Amiri-Kordestani L, Barac A, Beckman JA, Chism DD, et al. Cardio-oncology: vascular and metabolic perspectives: a scientific statement from the American Heart Association. Circulation 2019;139:e579602.

4. Gora-Tybor J, Medras E, Calbecka M, Kolkowska-Lesniak 
A, Ponikowska-Szyba E, Robak T, et al. Real-life comparison of severe vascular events and other non-hematological complications in patients with chronic myeloid leukemia undergoing second-line nilotinib or dasatinib treatment. Leuk Lymphoma 2015;56:2309-14.

5. Chai-Adisaksopha C, Lam W, Hillis C. Major arterial events in patients with chronic myeloid leukemia treated with tyrosine kinase inhibitors: a meta-analysis. Leuk Lymphoma 2016;57:1300-10.

6. Schlumberger M, Tahara M, Wirth LJ, Robinson B, Brose MS, Elisei R, et al. Lenvatinib versus placebo in radioiodine-refractory thyroid cancer. N Engl J Med 2015;372:62130.

7. Kim TD, Rea D, Schwarz M, Grille P, Nicolini FE, Rosti G, et al. Peripheral artery occlusive disease in chronic phase chronic myeloid leukemia patients treated with nilotinib or imatinib. Leukemia 2013;27:1316-21.

8. Zuo PY, Chen XL, Liu YW, Xiao CL, Liu CY. Increased risk of cerebrovascular events in patients with cancer treated with bevacizumab: a meta-analysis. PLoS One 2014;9:e102484.

9. Wang L, Huang Z, Huang W, Chen X, Shan P, Zhong P, et al. Inhibition of epidermal growth factor receptor attenuates atherosclerosis via decreasing inflammation and oxidative stress. Sci Rep 2017;8:45917.

10. Nonagase Y, Takeda M, Tanaka K, Hayashi H, Iwasa T, Nakagawa K. Treatment of EGFR mutation-positive non-small cell lung cancer complicated by Trousseau syndrome with gefitinib followed by osimertinib: a case report. Oncotarget 2018;9:29532-5. 